\title{
Gobernanza para el desarrollo territorial en el municipio de Florida - Valle del Cauca. Barrio El Progreso (comuna 3) y corregimiento de Tarragona 2000-2018
}

\section{Governance for territorial development in the municipality of Florida - Valle del Cauca. El Progreso neighborhood (commune 3) and Tarragona township 2000-2018}

\author{
Maria Arango \\ Docente, Universidad. Autónoma de Manizales, Colombia. \\ mariae.arango@autonoma.edu.co | 0000-0001-7775-9435 \\ Jose Julian Alzate \\ Docente, Universidad. Autónoma de Manizales, Colombia. \\ Jose.alzatef@autonoma.edu.co | 0000-0002-4316-0827
}

\begin{abstract}
Para citar este artículo: Arango, M., y Alzate, J. J (2020). Gobernanza para el desarrollo territorial en el municipio de Florida - Valle del Cauca. Barrio El Progreso (comuna 3) y corregimiento de Tarragona. 20002018. Entorno Geográfico, (20), 109-126. https://doi.org/10.25100/eg.v0i20.10850
\end{abstract}

\section{Resumen}

El artículo presenta los resultados de la investigación realizada en el barrio El Progreso y el corregimiento de Tarragona del municipio de Florida en el Valle del Cauca con el propósito de identificar la incidencia de la gobernanza en el desarrollo territorial de los sitios referidos. El ejercicio se realiza a partir de una metodología de tipo cualitativa que empleo el Marco Analítico de la GobernanzaMAG, el cual permitió establecer desde la interacción de sus actores los puntos nodales y validar las normas $\mathrm{u}$ acuerdos logrados entre estos, y como aportan al mejoramiento de las condiciones de vida de las poblaciones; como técnicas fueron utilizadas la entrevista semiestructurada a actores claves y el análisis documental tanto de informes de gestión y de documentos de política como los planes de desarrollo municipal como de referentes teóricos. Para el análisis de la información se utilizó el atlas ti. La técnica de triangulación de información fue útil al momento de contrarrestar la información obtenida de diferentes fuentes de información.

Como parte de los hallazgos se identificó que las interacciones entre los actores se dan como producto de la voluntad política, con un alto grado de liderazgo de algunos de ellos y en un escenario de innovación social que busca resolver los problemas de las comunidades, promoviendo una mayor cohesión social y en especial, el fortalecimiento de la sociedad civil; su capacidad de relacionamiento se identifica como factor clave en términos del cierre de brechas.

Palabras clave: Gobernanza, actores, desarrollo territorial, incidencia, cohesión social, Florida - departamento del Valle del Cauca - Colombia. 


\section{Abstract}

The article presents the results of the research carried out in the El Progreso neighborhood and the Tarragona district of the municipality of Florida in Valle del Cauca with the purpose of identifying the incidence of governance in the territorial development of the aforementioned territories. The exercise is carried out from a qualitative methodology that uses the Analytical Framework of GovernanceMAG, which allowed establishing the nodal points from the interaction of its actors and validating the norms or agreements reached between them, and how they contribute to the improvement of the living conditions of the populations; The techniques used were the semistructured interview with key actors and the documentary analysis of both management

Recibido: 21 de mayo de 2020

\section{Presentación}

La gobernanza como elemento catalizador del desarrollo de los territorios, aparece como un nuevo contrato social que permite la apropiación por parte de sus actores. La relación directa entre gobernanza y desarrollo territorial se evidencia a partir de un mejor desempeño de los indicadores de calidad de vida de las poblaciones en las diferentes escalas territoriales, producto, entre otras, de una prestación más eficiente de los servicios institucionales tanto públicos como privados, y de una mejor administración y gestión de los recursos.

El barrio El Progreso y el corregimiento de Tarragona, como parte del municipio de Florida, definidos como áreas de trabajo para la presente investigación, son reports and policy documents such as municipal development plans and theoretical references. For the information analysis the atlas ti was used. The information triangulation technique was useful when countering the information obtained from different information sources.

As part of the findings, it was identified that the interactions between the actors occur as a product of political will, with a high degree of leadership from some of them and in a scenario of social innovation that seeks to solve the problems of the communities, promoting a greater social cohesion and especially, the strengthening of civil society; Their ability to relate is identified as a key factor in terms of closing gaps.

Keywords: Governance, actors, territorial development, advocacy, social cohesion, department of Valle del Cauca - Colombia.

Aceptado: 21 de julio de 2020

escenarios de fenómenos de densificación territorial diferentes, asociados igualmente a diversas maneras de ver y gestionar el territorio para la satisfacción de las necesidades de sus pobladores en el marco del desarrollo económico y social. Ambos territorios son vulnerables socialmente; el barrio El Progreso, emerge de un proceso de consolidación territorial resultado de un desastre natural producto de la avalancha del rio Frayle en el año 1994 (Olvido, 2012); el corregimiento Tarragona, por su aislamiento territorial, estuvo al margen de los procesos de concertación e inversión social realizados entre 2000 y el 2018. 
2. Contexto del municipio de Florida Valle del Cauca

El municipio de Florida es uno de los 42 que integran el departamento del Valle del Cauca ubicado en la región pacífica - sur occidente colombiano como se reporta en el plano, con un área de $395 \mathrm{~km} 2$ y una población de 57.961 habitantes (DANE, 2018). El área urbana está dividida en cinco comunas con 32 barrios y la zona rural se compone de 12 corregimientos, 25 veredas, tres resguardos y dos cabildos indígenas; las áreas de trabajo seleccionadas son el barrio El Progreso y el corregimiento de Tarragona, territorios seleccionados, entre otros aspectos, por la cercanía de los investigadores con los mismos y su interés por identificar la incidencia de la gobernanza en los procesos del desarrollo territorial como alternativa de construcción social y de recuperación de la confianza del sistema de actores territoriales ante el descontento de sus habitantes por el registro reiterado de irregularidades en la gestión pública que debilitaron su confianza en la institucionalidad.
Por tanto, el desarrollo territorial se constituye en un gran desafío para el barrio El Progreso y el corregimiento de Tarragona, toda vez que en esencia comparten problemáticas sociales y económicas que dan cuenta de su complejidad, en términos de los retos para su desarrollo. Cabe señalar que ello dependerá de las decisiones y acciones que implementen sus agentes sociales y económicos, su institucionalidad y de cómo éstos interactúan en el marco de participación ciudadana.

Lograr esta articulación y acción conjunta entre los actores, sus recursos y capacidades, supone un análisis más detallado de las categorías que permiten determinar y comprender la incidencia de la gobernanza en el desarrollo territorial del municipio de Florida Valle del Cauca, el barrio El Progreso (Comuna 3) y el corregimiento de Tarragona, como posible mecanismo que contribuya a mejorar la toma de decisiones y su implementación en pro del desarrollo económico y social (Figura 1).

Fuente: Elaboración propia a partir de imágenes disponibles en la web/diciembre de 2017.

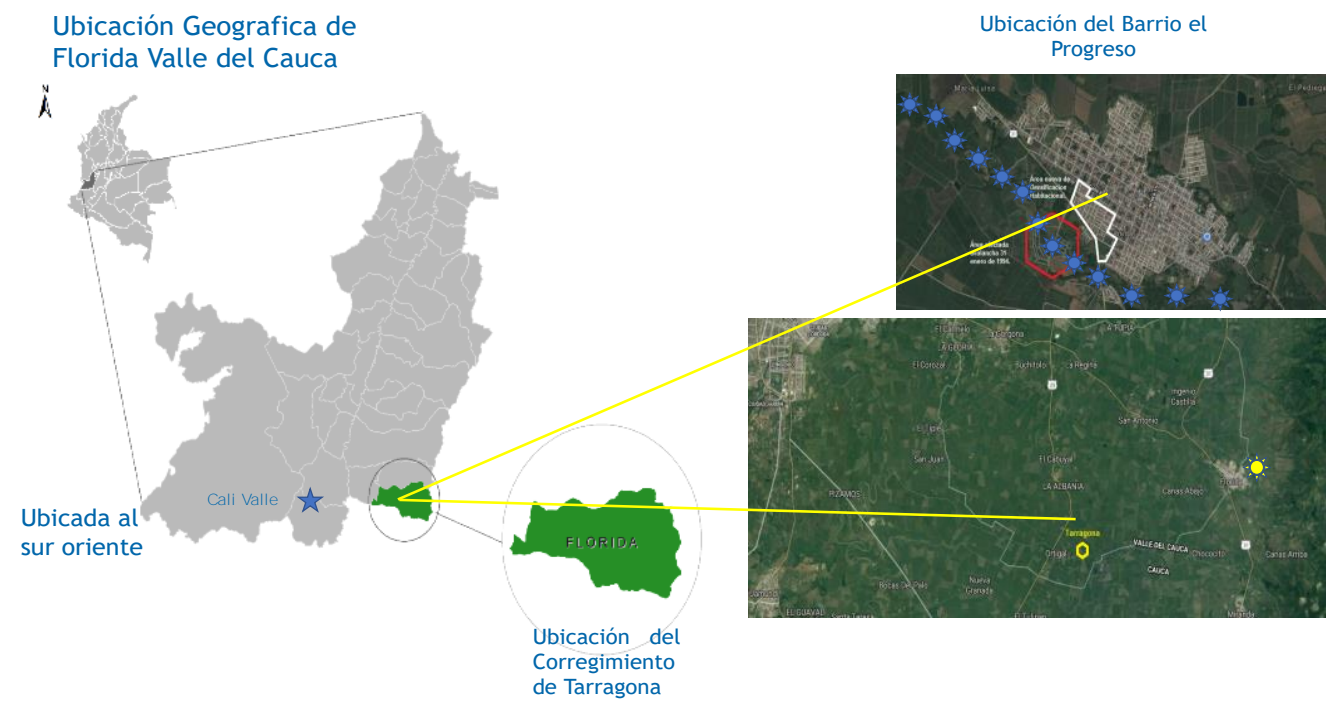

Figura 1. Ubicación geográfica del municipio de Florida - Valle del Cauca 
El desempeño municipal de Florida, medido por su Índice de Pobreza Multidimensional (IPM) reportado por el (DANE, 2020) para el municipio de Florida fue del $49 \%$, con un rezago de 21 puntos porcentuales frente al $70 \%$ del nivel nacional y con 9 puntos por encima del departamental registrado en un $40 \%$.
Respecto a la efectividad de la gestión desarrollada por el gobierno local, el Índice de Medición del Desempeño señala que, en el año 2017 (DANE, 2020), Florida estaba ubicado en un rango Medio Alto, con un puntaje de 49,2 puntos como se reporta en la Tabla 1.

Tabla 1. Resultados Medición de Desempeño Municipal Florida (MDM) general y por componente, 2017

\begin{tabular}{|c|c|c|c|c|c|}
\hline $\begin{array}{c}\text { Código } \\
\text { Dane }\end{array}$ & Departamento & Municipio & $\begin{array}{c}\text { Grupo de Capacidades } \\
\text { Iniciales }\end{array}$ & $\begin{array}{c}\text { Puntaje } \\
\text { MDM }\end{array}$ & $\begin{array}{c}\text { Puesto } \\
\text { Grupo } \\
\text { MDM }\end{array}$ \\
\hline 76275 & Valle del Cauca & Florida & G2-Nivel Medio Alto & 49,2 & 143 \\
\hline
\end{tabular}

\begin{tabular}{|c|c|c|c|c|c|c|c|c|c|}
\hline $\begin{array}{c}\text { Código } \\
\text { Dane }\end{array}$ & Departameto & Municipio & $\begin{array}{l}\text { Grupo de } \\
\text { Capacidades }\end{array}$ & \multicolumn{6}{|c|}{ Variables Municipales } \\
\hline \multirow{6}{*}{76275} & \multirow{6}{*}{$\begin{array}{l}\text { Valle del } \\
\text { Cauca }\end{array}$} & \multirow{6}{*}{ Florida } & \multirow{6}{*}{$\begin{array}{c}\text { G2-Nivel } \\
\text { Medio Alto }\end{array}$} & $\begin{array}{c}\text { Movilización } \\
\text { de Recursos } \\
\text { (Gestión) }\end{array}$ & $\begin{array}{c}\text { Ejecución } \\
\text { de Recursos } \\
\text { (Gestión) }\end{array}$ & $\begin{array}{c}\text { Ordenamiento } \\
\text { Territorial } \\
\text { (Gestión) }\end{array}$ & $\begin{array}{c}\text { Gobierno } \\
\text { Abierto y } \\
\text { Transparente } \\
\text { (Gestión) }\end{array}$ & $\begin{array}{c}\text { Puntaje } \\
\text { (Gestión) }\end{array}$ & $\begin{array}{c}\text { Puesto } \\
\text { Grupo } \\
\text { (Gestión) }\end{array}$ \\
\hline & & & & 23,2 & 60,7 & 26,4 & 85,8 & 49 & 141 \\
\hline & & & & $\begin{array}{c}\text { Educación } \\
\text { (Resultado) }\end{array}$ & $\begin{array}{c}\text { Servicios } \\
\text { (Resultados) }\end{array}$ & $\begin{array}{c}\text { Seguridad } \\
\text { (Resultados) }\end{array}$ & $\begin{array}{c}\text { Puntaje } \\
2016\end{array}$ & $\begin{array}{c}\text { Puntaje } \\
2017\end{array}$ & $\begin{array}{l}\text { Puesto } \\
\text { Grupo }\end{array}$ \\
\hline & & & & 52,2 & 63,6 & 88,3 & 74,2 & 74,6 & 14 \\
\hline & & & & $\begin{array}{c}\text { Movilización } \\
\text { de Recursos } \\
\text { (Gestión) }\end{array}$ & $\begin{array}{c}\text { Ejecución } \\
\text { de Recursos } \\
\text { (Gestión) }\end{array}$ & $\begin{array}{c}\text { Ordenamiento } \\
\text { Territorial } \\
\text { (Gestión) }\end{array}$ & $\begin{array}{c}\text { Gobierno } \\
\text { Abierto y } \\
\text { Transparente } \\
\text { (Gestión) }\end{array}$ & $\begin{array}{c}\text { Puntaje } \\
\text { (Gestión) }\end{array}$ & $\begin{array}{c}\text { Puesto } \\
\text { Grupo } \\
\text { (Gestión) }\end{array}$ \\
\hline & & & & 23,2 & 60,7 & 26,4 & 85,8 & 49 & 141 \\
\hline
\end{tabular}

Fuente: Departamento Nacional de Planeación. Medición de Desempeño Municipal 2017.

En materia de seguridad el DNP en el año 2017 reporta para el municipio un incremento de la tasa de homicidios por 100 habitantes entre los años 2008 - 2012: 2008 (53), 2009 (65), 2010 (74), 2011 (80) y 2012 (110), comparado con el departamento del Valle del Cauca con reportes de 2008 (70), 2009 (69), 2010 (78), 2011 (79) y 2012 (80) y el nivel nacional con tasas de 2008 (35), 2009 (36), 2010 (34), 2011 (35), 2012 (35) homicidios para el mismo periodo. Para el caso de Florida las cifras reflejan las condiciones de un territorio que ha estado bajo la influencia del conflicto armado, apalancado en alto grado por una ruta del narcotráfico estrechamente visible en el contexto nacional y las diferentes alianzas entre actores ilegales presentes en la región.

Frente a la gestión de los Ingresos Corrientes de Libre Destinación ICLD 
entre 2008 y 2016 a nivel del municipio se evidencia en términos generales y a pesar de las condiciones referidas presento un incremento del recaudo de ingresos propios (ingreso predial e industria y comercio), favoreciendo la ejecución de programas y proyectos de inversión social en favor de su población. Como se reporta en la Tabla 2.

Tabla 2. Relación de ICLD municipio de Florida - Valle del Cauca 2008 - 2016

\begin{tabular}{|l|l|}
\hline Año & Ingresos \\
\hline 2008 & $\$ 3.921 .711 .269$ \\
\hline 2009 & $\$ 4.962 .636 .157$ \\
\hline 2010 & $\$ 4.781 .397 .021$ \\
\hline 2011 & $\$ 4.258 .846 .751$ \\
\hline 2012 & $\$ 5.531 .570 .203$ \\
\hline 2013 & $\$ 5.919 .088 .108$ \\
\hline 2014 & $\$ 6.893 .569 .092$ \\
\hline 2015 & $\$ 7.941 .438 .454$ \\
\hline 2016 & $\$ 8.547 .803 .232$ \\
\hline
\end{tabular}

Fuente: Informes de Rendición de Cuentas Municipio de Florida, 2012 - 2016, Secretaría de Hacienda del año 2017 (Valle, 2017).

\section{Marco teórico de referencia}

El ejercicio investigativo se soporta principalmente en los aportes de Mazurek, autor de importantes obras como "Gobernabilidad y gobernanza de los territorios en América Latina" (Mazurek, 2009) y "Espacio y territorio. Instrumentos metodológicos de investigación social" (Mazurek, 2006). En segundo lugar, Luis Fernando Aguilar Villanueva desde sus aportes asociados a la gobernanza y la gestión pública (Aguilar, 2013; 2016). Y, en tercer lugar, se toman en cuenta los planteamientos de Marc Hufty, coeditor de libros como "¿A dónde va Bolivia? Gobernancia, gobernabilidad y democratización" (Hufty, 2005), "Movimientos sociales y ciudadanía" (Hufty, 2007) y el capítulo de libro "Una propuesta para concretar el concepto de gobernanza: El Marco Analítico de la Gobernanza" (Mazurek, 2009, p. 86).
La aproximación del territorio se aborda desde los postulados de (Monnet, 1999), quien lo asume como un espacio donde se desarrolla la acción humana y se estructuran las representaciones y significados del grupo social que lo habita, y lo complementa como con el de territorialidad, entendida como el conjunto de valores atribuidos a un territorio y la capacidad de un actor para ejercer una competencia sobre un espacio determinado. Desde esta perspectiva, el territorio apropiado se hace más complejo, y se transforma en un escenario de poder y de discusión, que busca entre otros, asegurar su supervivencia, construir viviendas $\mathrm{y}$ rutas, explotar productos y delimitar dominios (Capel, 2016).

La gobernanza aparece como una cualidad del sistema político-social, un nuevo estilo del gobierno estrechamente ligado a la 
cooperación, a la cohesión social, a la interacción entre el Estado y los actores no estatales (sociedad civil y mercado). Para el autor, en el marco de los procesos de descentralización del Estado, los actores de poder empiezan a gestarse al interior de los focos de decisión dados por las relaciones entre lo público y lo privado, generando cambios que afectan la territorialidad, actuando finalmente como cogobiernos o dinamizadores locales de poder que inciden de manera positiva o negativa sobre el territorio y las decisiones que allí se adopten. Para Hufty (Mazurek, 2009, p. 86):

"La gobernanza es una clase de hechos sociales y un objeto de estudio. «Gobernanza» se refiere a los procesos colectivos, formales tanto como informales, que determinan en una sociedad cómo se toman decisiones y se elaboran normas sociales con relación a asuntos públicos".

El autor plantea el Marco Analítico de la Gobernanza (MAG), como metodología que permite, desde la observación en el territorio, develar cómo se toman las decisiones formales e informales en torno a los problemas presentes en las comunidades y determinar el tipo de mediaciones que se dan entre los diferentes niveles de intervención de lo público y lo privado.

\section{Materiales y métodos}

Se utilizó la investigación cualitativa de tipo narrativo, descriptivo y exploratorio como estrategia metodológica que permitió para el periodo propuesto, determinar la dinámica de los procesos de gobernanza en el municipio de Florida- Valle del Cauca según lo acontecido en el barrio El Progreso y en el corregimiento de Tarragona. Con base en el MAG propuesto por Hufty (2007) fueron consideradas cinco categorías que permitieron comprender los procesos de gobernanza: los problemas; los actores; los puntos nodales; las normas; y los procesos.

Cada una de las categorías fueron analizadas a partir del uso de diferentes fuentes de información: Se emplearon como técnicas el análisis documental y la entrevista semiestructurada dirigida a diferentes actores, como líderes sociales y/o comunitarios e institucionales. El análisis de la información se dio a través del atlas ti, software utilizado para análisis cualitativo que permitió develar la capacidad de articulación, propósitos, motivaciones y demás factores relevantes contenidos en la pregunta de investigación en un escenario de gobernanza analítica. El ejercicio fue realizado en cuatro fases: i. Revisión bibliográfica; ii. Estructuración y validación del trabajo; iii. Trabajo de campo, que comprendió observación directa, entrevistas y análisis de fuentes secundarias de gestión y planificación territorial como planes, programas $\mathrm{y}$ proyectos y sus respectivos informes de gestión; y iv. Fase de sistematización y análisis de la información.

La población vinculada al proceso de las entrevistas semiestructuradas estuvo conformada por un grupo de actores seleccionadas a través de un muestreo no probabilístico por conveniencia, en el cual, resultaron de gran valor, los actores referenciados entre estos. Como criterios de inclusión de estos se definieron: su nivel de conocimiento e influencia en territorio y vinculación con diferentes sectores: autoridades locales, concejales en ejercicio, veeduría ciudadana, líderes comunitarios, 
exalcaldes, funcionario del gobierno identificados con los códigos que se nacional, y empresarios, 14 en total registran en la Tabla 3.

Tabla 3. Códigos utilizados identificación de actores

\begin{tabular}{|l|l|}
\hline Código & Tipos de entrevistados \\
\hline A1, A2, A3 & Autoridades locales Educativas \\
\hline C1, C2, C3 & Concejales en ejercicio \\
\hline EX - C1 & Exconcejal \\
\hline V1. & Veeduría ciudadana \\
\hline L1, L2, L3 & Líderes comunitarios \\
\hline Ex A. & Exalcaldes \\
\hline Fgn. & Figura de Contexto Nacional \\
\hline Emp. & Empresario \\
\hline
\end{tabular}

Se mantiene la confidencialidad de la información respetando los protocolos de bioética de la Universidad Autónoma de
Manizales UAM. La matriz de análisis se presenta en la Tabla 4.

Tabla 4. Guía clasificación por categorías y subcategorías

\begin{tabular}{|l|l|l|}
\hline Categoría de Gobernanza & $\begin{array}{l}\text { Categoría de } \\
\text { Incidencia }\end{array}$ & $\begin{array}{l}\text { Categoría de Desarrollo } \\
\text { Territorial }\end{array}$ \\
\hline subcategorías & subcategorías & subcategorías \\
Problemas & Intereses & Sentido de pertenencia \\
Los Actores & Empoderamiento - & Sinergias \\
Autoridad o Legitimidad & poder & Interacciones \\
Los Puntos Nodales & Participación & Legalidad \\
Articulación de Actores & Regulación & Inversión \\
Las Normas & Control & Cierre de brechas sociales, \\
Acuerdos & Modificación & calidad de vida y bienestar \\
Los Procesos & Validación & \\
Cooperación- Articulación & Garantías & \\
Cohesión social & & \\
Relevancia social, & & \\
económica y política & & \\
\hline
\end{tabular}

Fuente. Elaboración propia 


\section{Resultados}

\section{i. Gobernanza como categoría}

El ejercicio permitió identificar las diferentes interrelaciones adelantadas entre los actores, líderes y comunidad en general a fin de avanzar en los propósitos marcados, de esta manera se observó la prioridad y relevancia social dada a las relaciones que lograron mayor densidad y cohesión en los territorios por parte de estos; se observó como la categoría de la gobernanza asociada a los líderes y comunidad general, presenta un nivel de relacionamiento fuerte con las acciones liderada por los actores claves identificados por la base comunitaria, derivadas de su autoridad y legitimidad. Marcada por su credibilidad, estrechamente ligada a las demás categorías (incidencia y desarrollo territorial) que en conjunto contribuyeron a construir las dinámicas sociales propia de cada territorio con la inclusión de los mejores procesos y buenas prácticas.

La credibilidad que encierran las interacciones entre los actores estuvieron revestidas de autoridad y legitimidad fortalecidas por la normatividad (reglas de juego o decisiones) producto de los (acuerdos formales $e$ informales) alcanzados, de igual manera en menor proporción se observó una relación de aquellas densidades articuladas por los actores que apuntaron a la situación problemática a resolver, asociada a procesos en donde la comunidad prioriza los procesos con alta pertinencia social, legal, económica y política como se muestra en el Figura 2.

\section{Fuente: Elaboración propia a partir del software Atlas Ti}

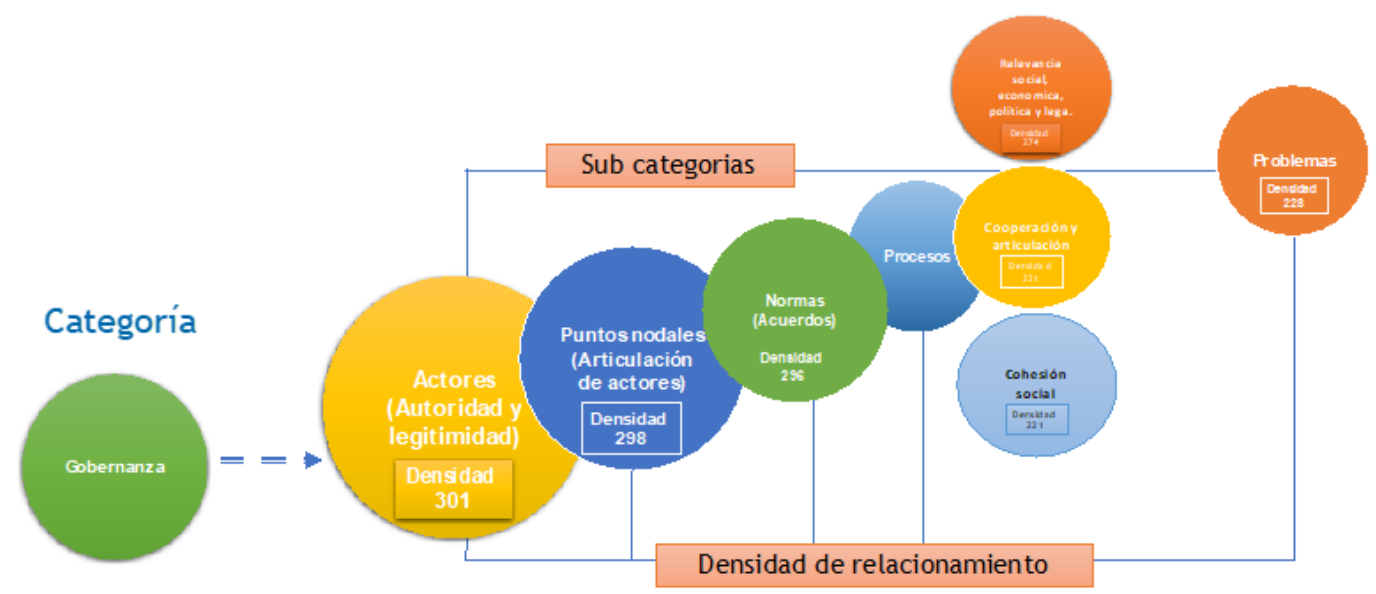

Figura 2. Gobernanza - densidad del relacionamiento

\section{ii. Incidencia como categoría}

Analizada la categoría de las incidencia ejercida en las dinámicas sociales desde la perspectiva de los actores, se determinó que la subcategoría con mayor densidad de relacionamiento es la voluntad política, asociada a la capacidad de dialogo con la institucionalidad en las diferentes escalas territoriales evidenciados en la creación de espacios de dialogo con el gobierno nacional, departamental y local, en especial con la alcaldía de Florida Valle como un factor de éxito; en menor proporción aparece la capacidad y densidad de 
relacionamiento asociada a temas de regulación, innovación social y modificación de los procesos por parte de los actores.

Para los actores, las motivaciones e intereses en esta categoría (incidencia) revelan que, en cualquiera de los territorios analizados, las prioridades y los intereses están relacionados a factores asociados con el involucramiento de las comunidades y el nivel de importancia que se derivan de sentirse proactivos a la hora de resolver una problemática, los espacios creados fueron claves, las mesas de dialogo, la interrelación y la negociación alcanzada en el caso particular con el actor con incidencia a nivel nacional fue valorada como una ventana a nuevos recursos para los procesos de negociación territorial desde la lógica de una mayor voluntad política de los actores del gobierno.

Para el caso del corregimiento de Tarragona se evidencia a partir de un actor político (concejal y exconcejal) y para el barrio El Progreso lo hace a través de líderes organizados. La Figura 3 evidencia el volumen de las densidades. La información detallada se reporta en la Figura 4, donde aparecen con mayor peso las subcategorías de voluntad política, garantías, confianza, participación y control.

Fuente: Elaboración propia a partir del software Atlas Ti

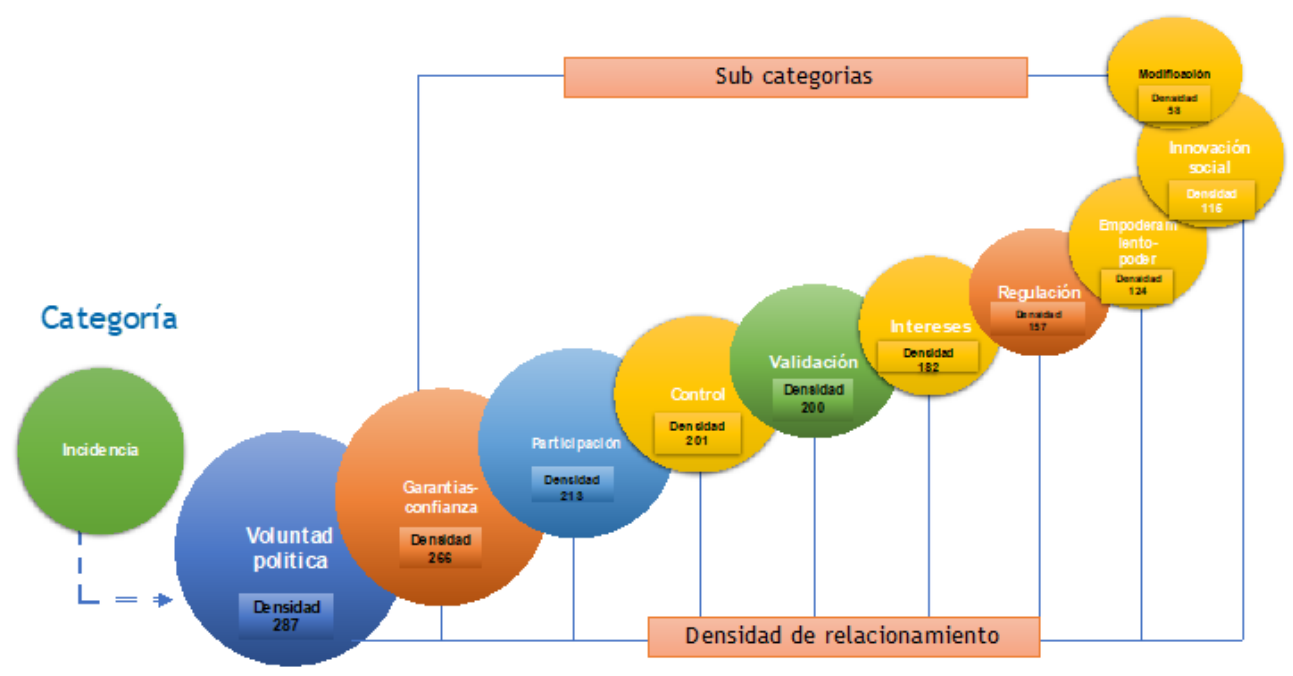

Figura 3. Incidencia - subcategorías y densidad del relacionamiento

\section{iii. Desarrollo territorial como categoría}

El cumplimiento de los acuerdos concertados se vuelve relevante a la hora de gestionar y avanzar en los procesos del desarrollo territorial; los actores entrevistados dieron relevancia a las intervenciones lideradas por los actores embebidas de voluntad política asociadas al direccionamiento territorial dado a los territorios, la manera cómo se gestionan los procesos de planeación territorial participativa y las sinergias logradas en términos de desarrollo (obras que dan solución a la problemática en cada territorio) bajo las premisas de la organización y la disciplina. 
Para el corregimiento de Tarragona se desarrolló una estrategia de visibilización territorial y política a través de actores institucionales con representatividad política que jalonaron en conjunto con la comunidad (junta de acción comunitaria) las principales obras a ejecutarse (saneamiento básico, recreación, deporte y cultura, seguridad) y su viabilidad; en menor proporción los actores valoran la forma como se gestionaron en forma oportuna los recursos asociado para el cierre de brechas sociales, calidad de vida y bienestar. Los líderes del barrio El Progreso, ratifican en sus aportes el valor que da la comunidad dio a su inclusión en los procesos de toma de decisiones $y$ construcción colectiva.

Fuente: Elaboración propia a partir del software Atlas Ti

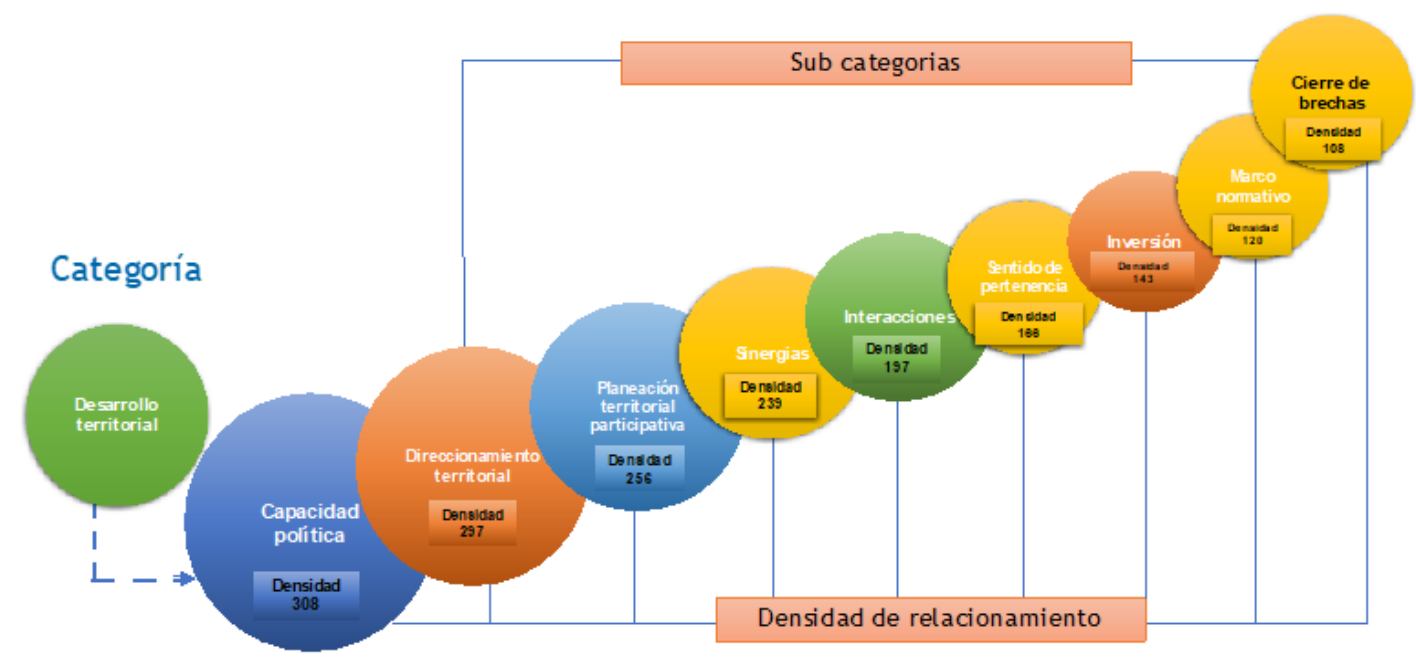

Figura 4. Desarrollo territorial - subcategorías - densidad del relacionamiento

Las mediaciones y relaciones motivadas por los actores dan cuenta que existe una fuerte densidad en el relacionamiento entre categorías - subcategorías. Resultan relevantes la participación, la voluntad política, las garantías, la validación y el control; las sinergias positivas están asociadas con la capacidad política, el direccionamiento y la planeación territorial participativa entre actores público, privado y sociedad civil en diferentes espacios de discusión local que consideran la importancia del contexto. Adicionalmente, se da valor a los procesos de inversión, legalidad y transparencia de las mediaciones en ambos territorios.
Reconocer los acuerdos pactados entre los actores, pasa por reconocer dos subcategorías interdependientes: la legalidad y la inversión asociadas con las intervenciones informales $\mathrm{o}$ formales desarrolladas en los territorios en respuesta a los diálogos entre los actores resulta determinante para mejorar el relacionamiento y potenciar la capacidad política asociada en términos del direccionamiento territorial y la apuesta de valor en los contextos municipal, departamental y nacional. La Figura 5 muestra el volumen o densidad del relacionamiento. 
Fuente: Elaboración propia a partir del software Atlas Ti

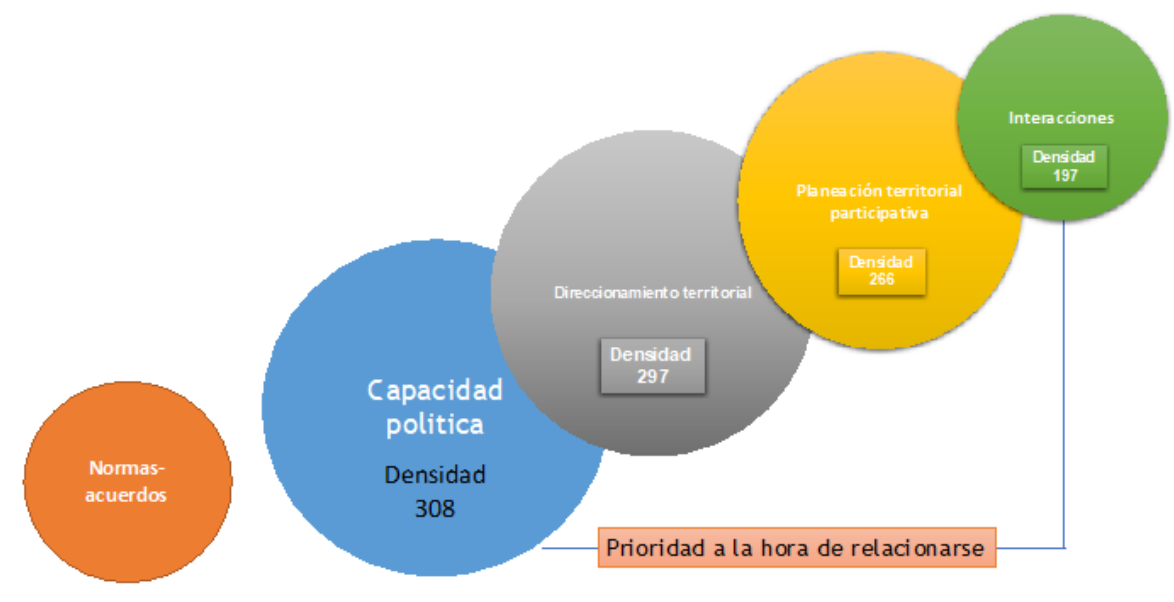

Figura 5. Densidad del relacionamiento a partir de los acuerdos

\section{Densidad del relacionamiento a partir} de la cohesión social

Los actores se relacionaron entre sí en los niveles nacional, departamental y local; la participación ciudadana como eje central del relacionamiento y del fortalecimiento de la cohesión social. Como subcategorías asociadas se identifican la capacidad política, la planeación territorial participativa, las sinergias y las interacciones como se presenta en la Figura 6, todas estas inscritas en procesos relevantes de tipo social, económico, legal y político permitiendo mejorar los procesos de cooperación y articulación con una red más densa de relaciones entre las subcategorías asociadas con la capacidad política, planeación territorial participativa, sinergias, interacciones $\mathrm{y}$ sentido de pertenencia como se presenta en la Figura 7. La posibilidad real del cierre de brechas sociales se vislumbra en un escenario de autoridad y legitimidad. 
Fuente: Elaboración propia a partir del software Atlas Ti

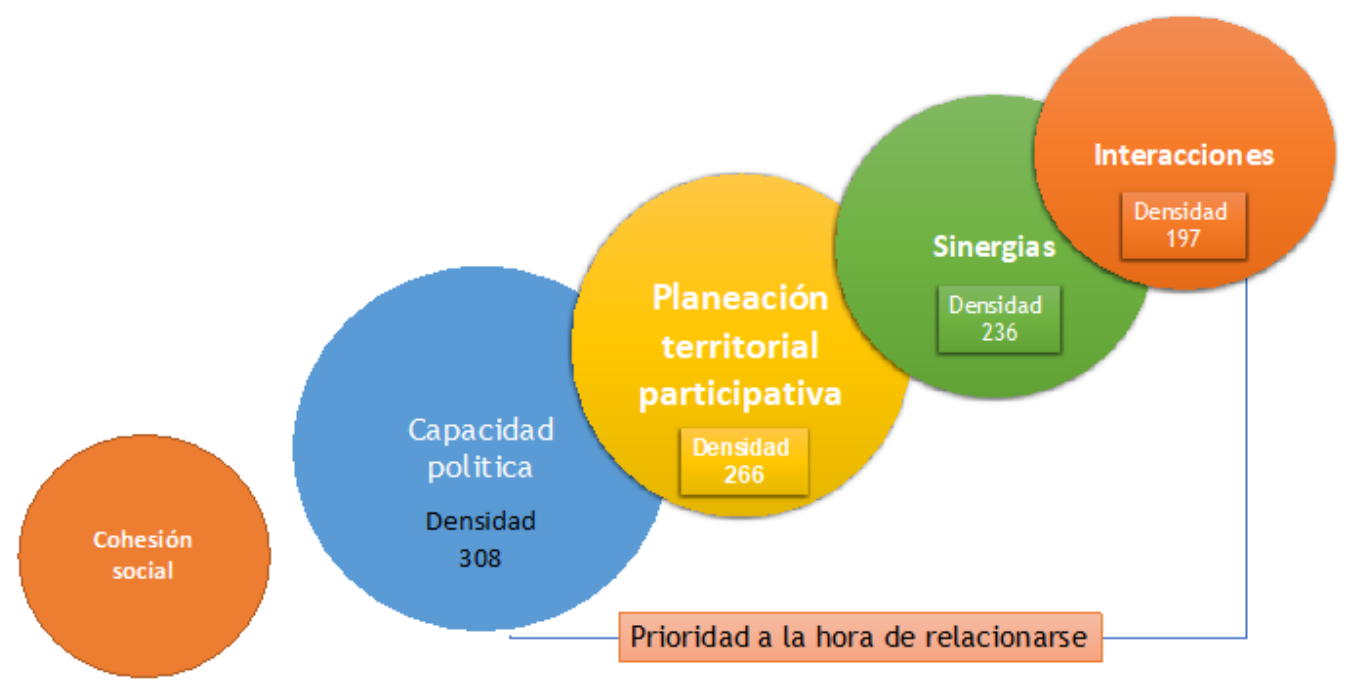

Figura 6. Densidad del relacionamiento a partir de la cohesión social

Fuente: Elaboración propia a partir del software Atlas Ti

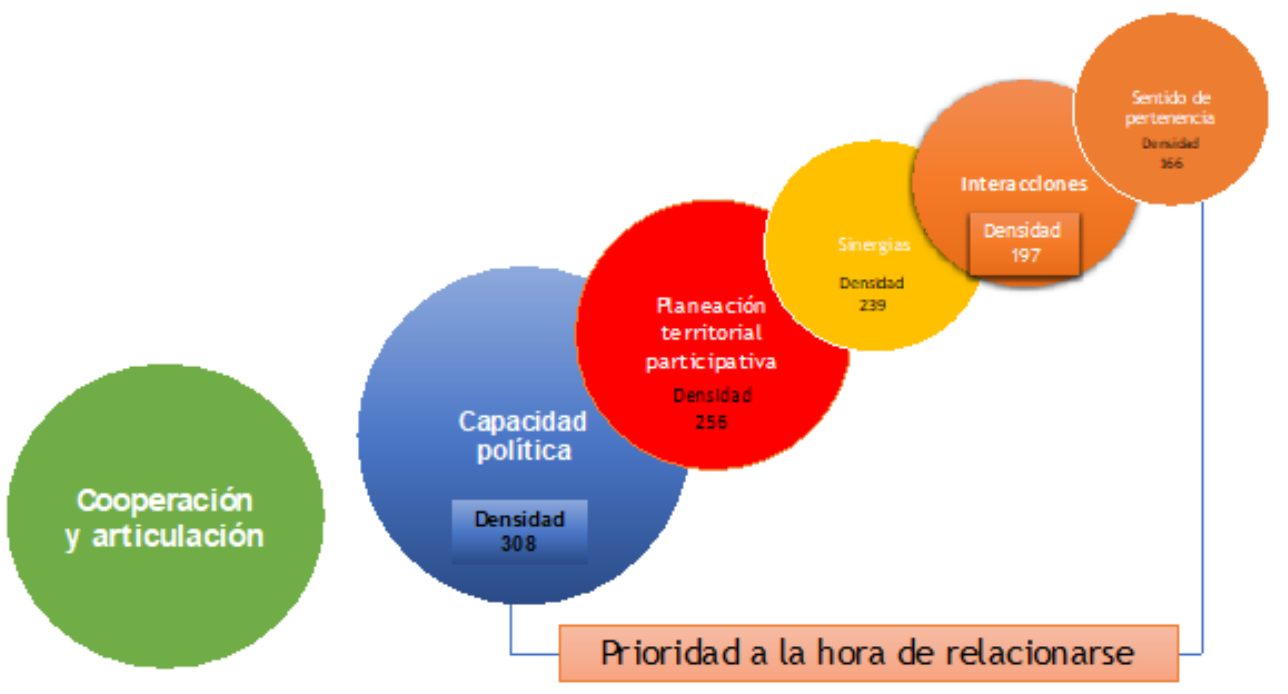

Figura 7. Densidad del relacionamiento a partir del interés de los actores

\section{Densidad de las relaciones de gobernanza asociada al desarrollo territorial}

Se logra identificar la importancia otorgada por los actores al momento de generar acuerdos, asociados a las buenas prácticas de direccionamiento territorial y a la planeación territorial participativa derivada de la buena gestión institucional de la administración municipal para ambos casos. La Figura 8 reporta la densidad de las relaciones, destacando la presencia de actores con autoridad y legitimidad que actúan a partir de acuerdos y normas establecidas, desde las cuales se direcciona 
la gestión territorial en presencia de voluntad política.

Fuente: Elaboración propia a partir del software Atlas Ti

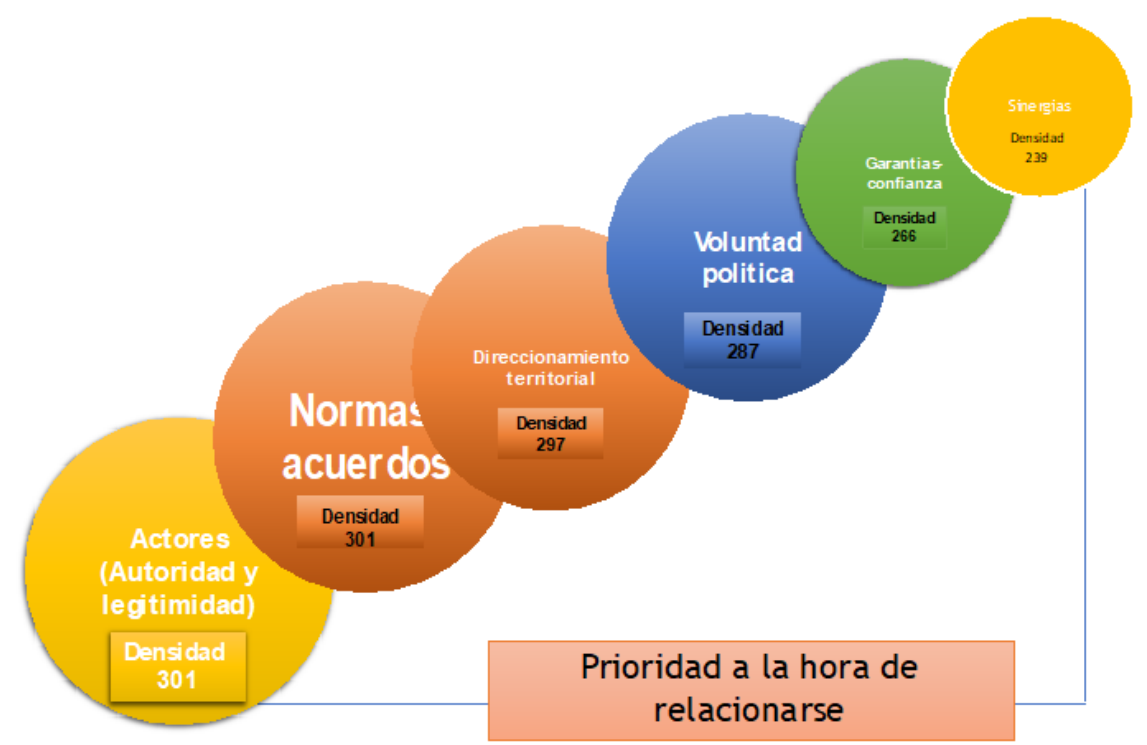

Figura 8. Densidad de las relaciones de gobernanza asociada al desarrollo territorial

\section{Análisis y discusión de los resultados}

Los hallazgos identificados a partir de la interacción con los actores clave permitieron validar la apuesta teórica de (Mazurek, 2009). en términos de que la gobernanza emerge como una cualidad del sistema político-social, un nuevo estilo del gobierno estrechamente ligado a la cooperación, a la cohesión social y a la interacción entre el Estado, la sociedad civil - comunidad y el mercado.

La innovación social permitió implementar un nuevo estilo de gobierno que permitió establecer una nueva ruta de trabajo fundamentado en el empoderamiento, la pertenencia y el compromiso de trabajo por la comunidad incluyendo el sentir y la acción colectiva a la hora de tomar las decisiones.

Para lograrlo, el corregimiento de Tarragona focalizó todo su trabajo a través de un actor institucional y político relevante con credibilidad, capacidad de liderazgo y depositario de la confianza de la comunidad, atributos que lo llevaron gracias a un proceso de voto popular a ser elegido concejal durante tres periodos consecutivos de gobierno entre el 2004 y el 2016. Su condición de actor político le permitió ser garante de los acuerdos, factor considerado como relevante en el MAG de referencia, donde aparecen en un nivel de mayor densidad los mecanismos usados por los actores para entender las características de su sistema político social, los mecanismos de adaptación al mismo y la forma de influir en los demás actores, a fin de cohesionar la comunidad y lograr los objetivos comunes. En el barrio El Progreso este rol fue identificado en un actor vinculado con el nivel central que permitió desde una apuesta multiactores y multiescalar como lo sugiere Cruz (2011), ser garante de los compromisos establecidos en una relación gana - gana "votos vs. obras ". Mejoramiento de los 
servicios públicos, construcción de viviendas, una escuela, un CAI, un jardín infantil y un polideportivo son parte de los beneficios recibidos por las comunidades $\mathrm{y}$ que apalancaron la consolidación territorial y el mejoramiento de las condiciones de vida de sus habitantes como resultado de lo que define Aguilar (2013) como gobernanza o el trabajo compartido para la toma de decisiones concertadas entre lo público y lo privado. Aparecen además de la institucionalidad publica actores como el Ingenio del Cauca y Trapiche Palestina reconocidos por su aporte en la generación de nuevos_empleos y su importancia en el proceso de cierre de brechas.

La presencia en los dos territorios de un actor con gran incidencia en el ámbito nacional permitió la visibilizarían de la comunidad frente a los actores del gobierno, como producto de los términos de negociación focalizados. Para Boisier (2012), uno de los atributos del Estado contemporáneo, es el de la inteligencia territorial, en el sentido de adapta su propia estructura sistémica territorial a las circunstancias y comprende las interacciones entre objetivos a nivel nacional, departamental y local, postulado que Cruz (2011), avala al privilegiar como ya se ha referido las relaciones dadas en las diferentes escalas, la base de negociación comunitaria y los actores claves a la hora de establecer vínculos y formas de asociatividad para gestionar los acuerdos y generar sostenibilidad de las acciones a través de nuevas fuentes de financiación que involucren la institucionalidad y capacidad política del gobierno nacional con la voluntad política de los alcaldes y demás actores del gobierno y su capacidad de agencia.

\section{i. Los actores}

Estos fueron clasificados según su gestión en el territorio. Algunos para el caso del barrio El Progreso se remontaron a su origen como Acuavalle, la Corporación Autónoma Regional del Valle del Cauca CVC e Ingeominas, todos estos ayudaron con la consolidación territorial en alianza con la institucionalidad púbica del orden nacional y regional, la administración municipal y sus secretarias, y los actores de base comunitaria, entre ellos las Juntas de Acción Comunal, las veedurías ciudadanas y los grupos organizados. Como videncia del trabajo colaborativo reconocen la construcción de obras como una escuela, un Centro de Atención Inmediata (CAI) para la policía, el mejoramiento y construcción de red de alcantarillado, una biblioteca y un polideportivo.

Para el caso del corregimiento de Tarragona se reconoce como el principal logro la consolidación de un proceso comunitario liderado por un actor relevante, posteriormente elegido concejal entre los años 2004-2018. Se identifico un esquema de trabajo que resignifica el rol de la participación comunitaria y de los actores institucionales locales sin detrimento de las mediaciones con otros actores relevantes del contexto local, departamental y nacional.

\section{ii. Las normas - los acuerdos}

Desde el MAG se logra identificar los acuerdos formales e informales entre los actores y que dan origen al proceso de consolidación territorial 2000-2018. Se resalta para el barrio El Progreso, como a pesar de la existencia de un acuerdo formal de urgencia manifiesta en respuesta a la avalancha que da origen al barrio por parte de INGEOMINAS, en el cual se determina 
que la población estaba localizada en una zona de alto riesgo y sus restricciones para otorgar licencias de urbanismo y de construcción, se privilegia la necesidad de los damnificados; a pesar de lo expuesto, se logra consolidar el barrio y posteriormente la comuna 3 .

Para el caso del corregimiento de Tarragona, los acuerdos y normas se definieron en el marco de una estrategia se innovación social que permitió a través de un proceso de elección popular legitimar un actor comunitario como actor político y sostenerlo en este escenario como concejal para el periodo 2004 - 2016. Desde este escenario político logra incorporar en la agenda municipal las necesidades de la población y la consolidación de acuerdos formales entre estos, el acuerdo suscrito con un actor del nivel nacional definido en clave "votos vs obras" y otros que fueron materializados en los planes de desarrollo correspondientes al periodo 2000-2018", desde los cuales se gestionaron procesos articulados con el gobierno nacional $\mathrm{y}$ departamental.

\section{iii. Los puntos nodales o escenarios de concertación}

Para el barrio El Progreso entre los años 2000 - 2018 se establecen por parte de los actores los siguientes espacios de concertación o "diálogos institucionales":

La mesa institucional urgencia manifiesta con presencia de actores de nivel nacional, departamental y local, la mesa de trabajo permanente junta de vivienda comunitaria El Progreso, la mesa de seguridad del programa territorios seguros y en paz -MONPAZ-, la mesa de diálogo "comunidad y juntas de acción de los barrios consolidados", espacios que lograron la articulación de la población clave incrementando la densidad de las relaciones en función de sus intereses compartidos. Se destaca en este apartado, la "mesa de gobierno" desde la cual, según los testimonios de los actores, se logra incluir a otros actores considerados críticos en el proceso, representado en pandilleros y expandilleros que desde sus normas logran establecer otro tipo de acuerdos en el territorio que generaron miedo $\mathrm{e}$ incertidumbre en la comunidad.

Para el corregimiento de Tarragona para el mismo periodo, se identifican los siguientes puntos nodales: los diálogos institucionales, la mesa de articulación institucional como una instancia de discusión y seguimiento a los acuerdos pactados con los actores nacionales y departamentales en representación de la institucionalidad. Desde la iniciativa comunitaria se identifican tres instancias: espacios de diálogo permanente, espacios de dialogo con los actores políticos y mesa de acuerdo de voluntad política con el actor del nivel nacional bajo la prensa "votos vs obras, a partir de la cual se logran materializar obras de beneficio comunitario como el mejoramiento de los servicios públicos y la construcción de infraestructura educativa, deportiva $\mathrm{y}$ cultural.

\section{Articulación de los hallazgos para la definición de la estrategia de gobernanza local para el municipio de Florida}

La estrategia para la gobernanza local según los hallazgos de la investigación y el nivel de concurrencia de las subcategorías deberá incorporar de manera explícita en la estrategia de gobernanza local los ejes articuladores reportados en la Figura 9, donde se consideran las dos nuevas subcategorías emergentes enunciadas: 
innovación social y la voluntad política

(Tabla 5).

Fuente: Elaboración propia a partir de ejercicio de investigación

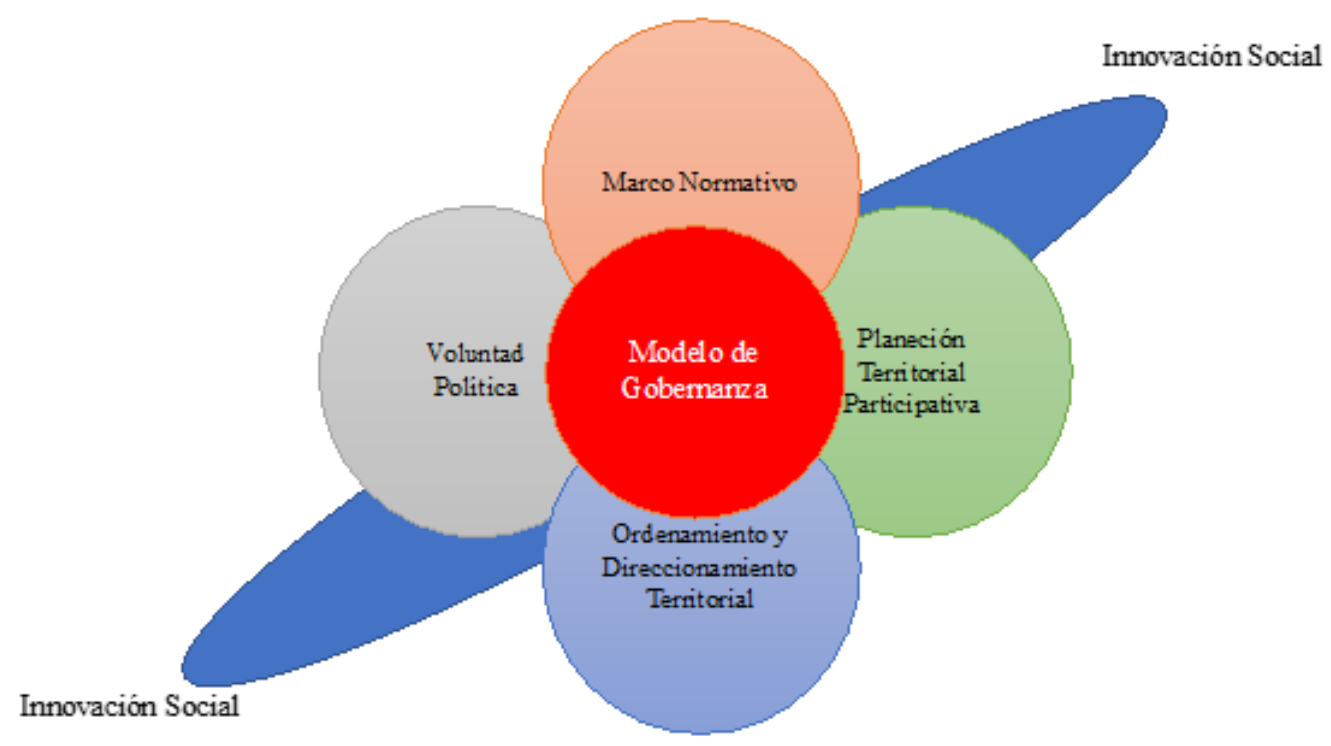

Figura 9. Estrategia de Gobernanza

Tabla 5. Categorías y subcategorías vinculadas a la estrategia de gobernanza para Florida

\begin{tabular}{|c|c|}
\hline Categoría principal & Subcategorías \\
\hline \multirow[t]{4}{*}{ Desarrollo Territorial } & $\begin{array}{l}\text { - Planeación Territorial Participativa: Se } \\
\text { Relaciona } 256 \text { Veces. }\end{array}$ \\
\hline & $\begin{array}{lll}\text { - Legalidad- Marco } & \text { Normativo: } & \text { Se } \\
\text { Relaciona } 120 \text { Veces. } & & \end{array}$ \\
\hline & $\begin{array}{l}\text { - Capacidad Política: Se Relaciona } 308 \\
\text { Veces. }\end{array}$ \\
\hline & $\begin{array}{lll}\text { - Direccionamiento } & \text { Territorial: } & \text { Se } \\
\text { Relaciona } 297 \text { Veces. } & & \end{array}$ \\
\hline Categoría principal & Subcategorías \\
\hline Incidencia & $\begin{array}{l}\text { - Voluntad Política: Se Relaciona } 287 \text { Veces } \\
\text { - Innovación Social: Se Relaciona } 115 \text { Veces }\end{array}$ \\
\hline
\end{tabular}

Fuente: Elaboración propia. 


\section{i. Marco normativo y de legalidad}

Este componte es clave a la hora de propiciar la participación ciudadana en los procesos de regulación y control de las acciones públicas y la promoción de las sinergias entre actores, fuertemente asociada con los conceptos de la legalidad. El componente normativo blindará los procesos en términos sociales, políticos, económicos entre otros. Propiciando espacios a los actores.

\section{ii. Planeación territorial participativa}

Permitirá a los actores disponer de las condiciones apropiadas para generar todas las interacciones y las acciones necesarias para logra las transformaciones requeridas en los territorios, considerando el sistema de actores territoriales para garantizar acuerdos viables y efectivos.

\section{iii. Ordenamiento y direccionamiento territorial}

El gobierno local, a través de este proceso y en concordancia con las necesidades de los

\section{Referencias bibliográficas}

Aguilar, L. F. (2013). El gobierno del gobierno. Toluca, México: Instituto Nacional de Administración Pública, A.C.

Aguilar, L. F. (2016). Democracia, gobernabilidad y gobernanza. México: Instituto Nacional Electoral.

Boisier, S. (2012). BOISIER Reflexiones Sobre Los Procesos Territoriales en El Siglo XXI. Huellas en El Territorio y Trazos en El Mapa. Madrid, España: Editorial Académica Española.

Capel, H. (2016). Las ciencias sociales y el estudio del territorio. Revista Bibliográfica de Geografía y actores, formulara e implementara desde los lineamientos normativos los instrumentos de gestión y planificación que le permitirán desde la visión compartida avanzar en el desarrollo territorial en respuesta a las demandas de sus habitantes en el corto, mediano y largo plazo.

\section{iv. Voluntad política}

Toda intervención territorial, debe tener voluntad de las partes a fin de cohesionar a todos los actores, generar empoderamiento, propiciar la innovación social y avanzar en el cierre de brechas sociales.

\section{v. Innovación social}

En el marco de las acciones de los actores, la pertinencia de la creatividad y la transformación social como resultado de la innovación social se reconoce como un eje transversal de la estrategia; desde un ejercicio inclusivo permitirá retroalimentar los aprendizajes y procesos en un territorio en constante aprendizaje.

Ciencias Sociales, 21(1149), s.p. https://www.raco.cat/index.php/Bib lio3w/article/view/305885

Cruz, B. N. (2011). Soportes teóricos y etnográficos sobre conceptos de territorio. Co-herencia, 8(14), 209229.

Departamento Administrativo Nacional de Estadística. DANE. (2018). Florida Valle. Bogotá, Colombia: TerridataSistema de Estadísticas Territoriales.

Departamento Administrativo Nacional de Estadística. DANE. $(27$ de septiembre de 2020). Índice de Desempeño Municipal. Recuperado de https://bit.ly/3fO5vhG 
Hufty, M. (2005). ¿A dónde va Bolivia? Gobernancia, Gobernabilidad $y$ Democratización. Bolivia: Plural Editores.

Hutfy, M. (2007). Movimientos sociales y ciudadanía. Bolivia: Plural Editores.

Mazurek, H. (2006). Espacio y territorio Instrumentos metodológicos de investigación social. La Paz: xEstratégica en Bolivia.

Mazurek, H. (2009). Gobernabilidad y bobernanza de los territorios de América Latina. Lima, Perú: Instituto Francés de Estudios Andinos.

Monnet, J. (1999). Globalización y territorializaciones areolar $y$ reticular: Los Ángeles y la Ciudad de México. Toluca, México: Instituto universitario de Francia / Universidad de Toulouse-Le Mirail. Olvido, L. (11 de julio de 2012). La Cola de Rata. Recuperado de https://bit.ly/33tvCFH

Valle, M. D. (2017). Informe de gestión. Florida, Valle: Alcaldía de Florida, Valle (Colombia). 